\title{
OFDM Wireless Downhole Transmission Systems and Proposed SLM Method for PAPR Reduction
}

\author{
Renze Luo ${ }^{1}$, Yupu Dang ${ }^{2}$, Rui Li ${ }^{1}$, Jiao Yang ${ }^{1}$ \\ ${ }^{1}$ State Key Laboratory of Oil and Gas Reservoir Geology and Exploitation, Southwest Petroleum University, Chengdu, China \\ ${ }^{2}$ Department of Well Tech, China Oilfield Services Limited, Beijing, China \\ Email: lrzsmith@126.com
}

Received September 13, 2013; revised October 13, 2013; accepted October 20, 2013

Copyright (C) 2014 Renze Luo et al. This is an open access article distributed under the Creative Commons Attribution License, which permits unrestricted use, distribution, and reproduction in any medium, provided the original work is properly cited. In accordance of the Creative Commons Attribution License all Copyrights (c) 2014 are reserved for SCIRP and the owner of the intellectual property Renze Luo et al. All Copyright (C 2014 are guarded by law and by SCIRP as a guardian.

\begin{abstract}
At present, mud pulse transmission systems are widely used in downhole data transmission. But the systems are very low in transmission efficiency, only 5 - 10 bits/s, with very large anti-inter-symbol-interference (ISI). It cannot meet high requirements for high-speed transmission of modern logging system. The development of communication technology has laid some foundation for this requirement. For this purpose, the Orthogonal Frequency Division Multiplexing (OFDM) Wireless Downhole Transmission Systems are proposed for the first time because of their high transmission rate, anti-inter-symbol-interference (ISI), and high spectral efficiency, etc. Due to non-linear power amplifier (PA) of logging systems with limited dynamic range, the drawbacks of high peak-average power ratio (PAPR) may outweigh all the potential benefits of OFDM wireless downhole transmission systems. Selective mapping (SLM) method can reduce the PAPR of OFDM logging signals without distortion. But at the receiver, the conventional SLM method needs exact bits of side information (SI) to recover the data signal. The probability of erroneous SI detection has a significant influence on the error performance of the system. And individual transmissions of SI result in the reduction of bandwidth efficiency. To restore the exact data signal, our scheme codes the SI bits by linear block codes (LBC), and is easily decoded by syndrome decoding. And then the coding SI bits are superimposed onto the logging signals to omit SI bits transmission. The theory and simulation results show that the proposed method has better performance than the conventional one. Accordingly, the OFDM wireless downhole transmission systems can tackle the high PAPR problem, and highten the transmission rate of logging signals.
\end{abstract}

\section{KEYWORDS}

Orthogonal Frequency Division Multiplexing Wireless Downhole Transmission Systems; Orthogonal Frequency Division Multiplexing (OFDM); Logging While Drilling (LWD); Peak-to-Average Power Ratio (PAPR); Selected Mapping (SLM)

\section{Introduction}

Nowadays, due to limited data transfer rate of mud pulse transmission [1-3], no logging data can be transmitted real-timely to the ground. Schumlburger Company owns technology of pulse transmission instruments more mature than that of other companies, increasing the transmission efficiency to 5 - 10 bit/s. But they only provide service but not device. As a consequence, the high-speed and efficient transmission of downhole signals has been the bottleneck in the development of downhole transmis- sion technology. The Orthogonal Frequency Division Multiplexing (OFDM) is one of the core technologies for the fourth generation (4G) mobile communication systems with high transmission rate, high spectral efficiency, frequency selective fading tolerance due to multi-path, immunity to the frequency selective fading channels, and power efficiency [4,5]. Consequently, OFDM technology has been applied to the downhole wireline transmission system [6], such as LOGIQ logging system of Halliburton [7], network LEAP800 logging system [8,9], downhole OFDM communication systems of Baker Hughes in 
patent [10], remote wireless transmission [11-13], etc. These systems improve the downhole transmission rate up to Megabit. They can overcome the drawbacks of the traditional logging transmission system, such as low transmission rate, poor efficiency and strong inter-symbol-interference (ISI), etc. Meanwhile, wireline systems [14-16] have poor stability of connection and complicated structure.

From the above, the OFDM wireless downhole transmission systems will meet the high-speed transmission challenge in the existing logging systems. But the systems are inefficient in power because of the high peakto-average power ratio (PAPR) inherent in OFDM signals. High PAPR values yield significant in-band distortion and undesired out-of-band radiation when it gets through a non-linear power amplifier (PA). Many schemes have been proposed to reduce PAPR, such as companding technique [17], clipping [18,19], partial transmit sequence (PTS) [20,21], Code Blocking [22-24], tone reservation (TR) scheme [25], and selective mapping (SLM) [26-29]. Among these schemes, SLM is more popular than others because it reduces the high PAPR of OFDM signal without distortion. However, the transmission of side information is one of the main drawbacks of SLM.

In this case, this paper proposes OFDM wireless downhole transmission systems and an improved SLM scheme to reduce PAPR. The proposed SLM method will sacrifice a little computation because of coding, but save bandwidth and improve error performance of systems. Consequently, the systems will make a good performance on high rate downhole transmission.

\section{Novel OFDM Wireless Downhole Transmission Systems}

The novel OFDM wireless downhole transmission systems are shown as Figure 1. In the systems, waveforms $\left\{s_{n}(t)\right\}$ are acquired by measuring devices (like sensors), and transmitted to a remote receiver for further control. The signals $\left\{s_{n}(t)\right\}$ denote the original downhole signals. The bit sequences are obtained through analog to digital converter (ADC). Then OFDM modulations are carried out for these parallel bit sequences at OFDM transmitter. After the processed information passes through complicated downhole channel, the OFDM receiver does the inverse steps to restore the logging data $\left\{s_{n}(t)\right\}$.

The OFDM wireless downhole transmission systems can reach a transmission rate of megabit per second. The transmission speed is much higher than that of the existing LWD transmission technology. Although OFDM has achieved great progress, the research on OFDM wireless downhole transmission systems have not been conducted by anyone so far. Once the systems are applied, they will further promote the development of high-speed and realtime downhole transmission technology. The main advantages of the novel OFDM wireless downhole transmission systems are:

- High-speed transmission up to megabits per second or more.

- The immunity to the frequency selective fading channels.

- High spectral efficiency.

- Easy installation.

\section{Proposed SLM Scheme}

In the systems, all the downhole logging signals are demodulated onto the carriers which are orthogonal to each other. If $\mathrm{N}$ sub-carriers are used, and each sub-carrier is modulated using logging symbols, the OFDM logging symbol $s(t)$ is expressed as

$$
s(t)=\frac{1}{\sqrt{N}} \sum_{n=0}^{N-1} S_{n} \mathrm{e}^{j 2 \pi \frac{n}{N} t}, 0 \leq t \leq N-1
$$

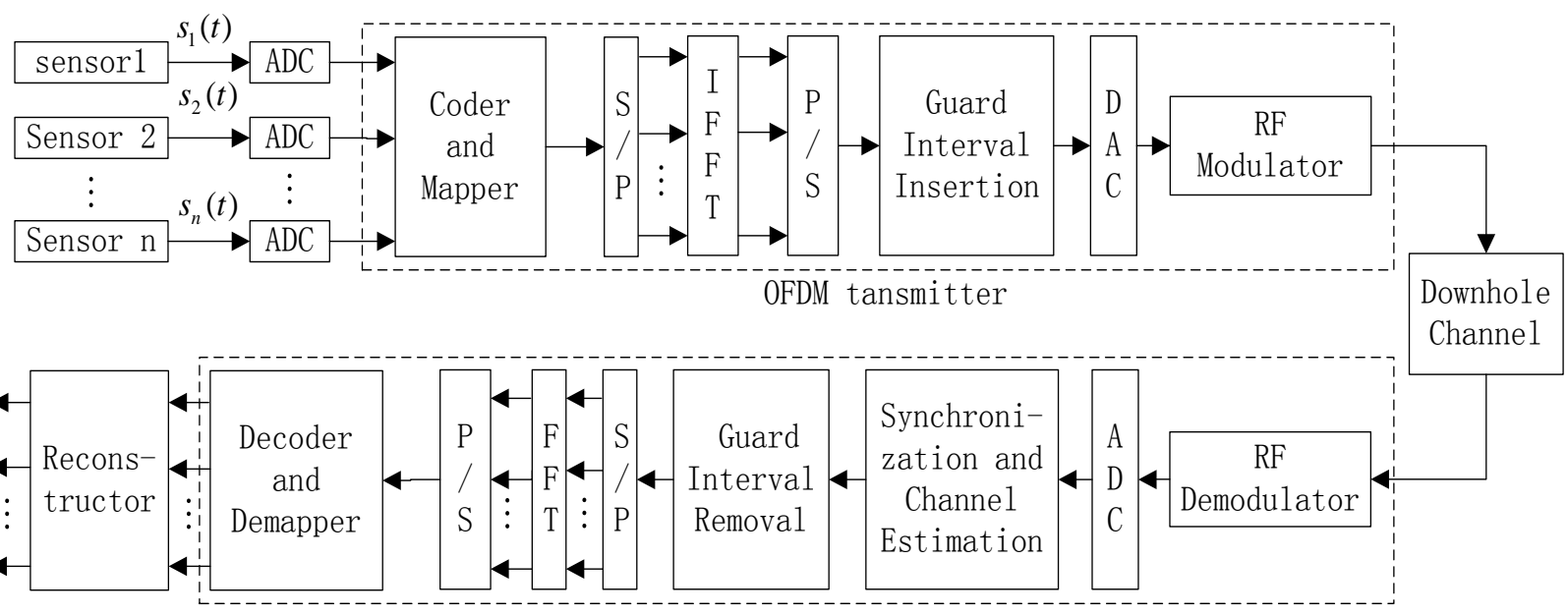

OFDM receiver

Figure 1. The block diagram of proposed OFDM wireless downhole transmission systems. 
where $S_{n}$ are the logging symbols, and $N$ is the number of sub-carriers.

The PAPR of an OFDM logging signal is written as

$$
P A P R \triangleq \frac{\max _{0 \leq t \leq N-1}|s(t)|^{2}}{E\left\{|s(t)|^{2}\right\}}
$$

where $E[\cdot]$ denotes the expected value.

The novel OFDM wireless downhole transmission systems have high PAPR, which requires linear, large-dynamic-range amplifiers that are inefficient and expensive to operate. This paper focuses on improving traditional SLM scheme which reduced PAPR without distortion.

The traditional SLM scheme requires the transmission of several side information (SI) bits for each data block. SI bits are the indexes of the selected vector to be transmitted. SI bits are generally transmitted as a set of log2 $(U)$ bits. $U$ is the number of phase sequences. The probability of erroneous SI detection has a significant influence on the error performance of the system. If the receiver does not detect the correct SI index, the whole data block will be lost each time. If the complexity is taken into consideration, the number of phase sequences is $U=8$. Therefore it needs $3 \mathrm{SI}$ bits to mark which vector is selected. In practice, a channel code must be used to protect the SI bits. In this paper, the linear block codes are used because of its easy implementation.

The Proposed SLM method is shown in Figure 2. The logging signals are gathered by the downhole sensors. After the serial transforming to parallel signals, the symbol sequences multiply the phase sequences $S_{u}=\left[S_{0}, S_{1}, \cdots, S_{U}\right], 1 \leq u \leq U$ to generate alternative logging symbol sequences $X_{u}=\left[X_{0}, X_{1}, \cdots, X_{U}\right]$.

Before IFFT, the SI bits are coded by linear block codes (LBC). Linear block codes (LBC) are a class of parity-check codes that are described as $(n, k)$. The encoder transforms a block of $\mathrm{k}$ message digits (a message vector) into a longer block of $n$ codeword digits (a code vector) constructed from a given alphabet of elements $[4,5]$. Here, Take a $(6,3)$ code for SI coding. It has $2^{k}=2^{3}=8$ message vectors, and therefore 8 codeword.

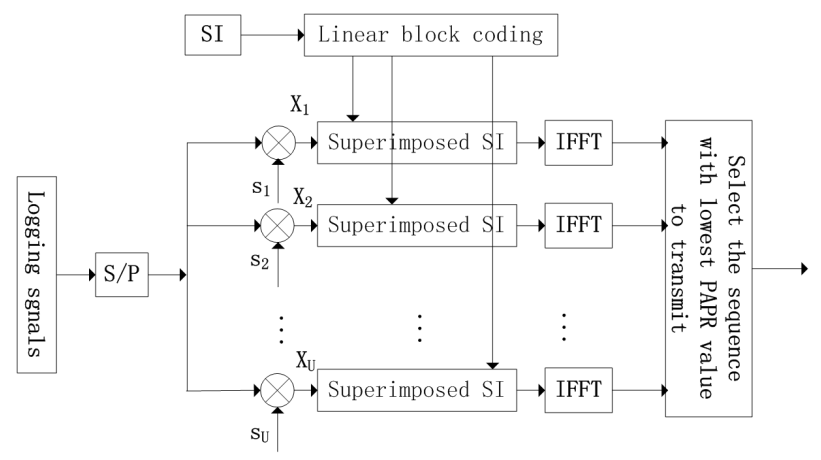

Figure 2. Proposed SLM method.
It is easy to obtain a 6-bit codeword mapping table as is shown in Table 1.

This mapping can also be indicated in Equation (3). That is, each of the set of $2^{3}$ codeword $\{\boldsymbol{U}\}$ is described by

$$
\boldsymbol{U}=\boldsymbol{m} \boldsymbol{G}
$$

where $\boldsymbol{m}$ is the massage vector, a sequence of $\mathrm{k}$ message bits $(1 \times 3$ matrix $)$. $\boldsymbol{G}$ is the generator matrix defined as the following $3 \times 6$ array:

$$
\boldsymbol{G}=\left[\begin{array}{l}
\boldsymbol{V}_{1} \\
\boldsymbol{V}_{2} \\
\boldsymbol{V}_{3}
\end{array}\right]=\left[\begin{array}{llllll}
1 & 1 & 0 & 1 & 0 & 0 \\
0 & 1 & 1 & 0 & 1 & 0 \\
1 & 0 & 1 & 0 & 0 & 1
\end{array}\right]=\left[\boldsymbol{P} \mid \boldsymbol{I}_{\boldsymbol{k}}\right]
$$

where $\boldsymbol{I}_{k}$ is a $k \times k$ unit matrix, $\boldsymbol{P}$ is a $k \times(n-k)$ matrix. First $k$ code is parity check bit to provide redundancy against errors. And the following $n-k$ code is message sequence.

The corresponding error correction decoding using syndrome test and error correction was introduced in [30]. With the parity-check matrix $\boldsymbol{H}$, it can decode the received vectors. For $(6,3)$ generator matrix $\boldsymbol{G}$, the corresponding matrix $\boldsymbol{H}$ is

$$
\boldsymbol{H}=\left[\begin{array}{llllll}
1 & 0 & 0 & 1 & 0 & 1 \\
0 & 1 & 0 & 1 & 1 & 0 \\
0 & 0 & 1 & 0 & 1 & 1
\end{array}\right]
$$

As long as the parity-check matrix $\boldsymbol{H}$ is given, the relationship between parity check bit and information bits is completely confirmed in coding.

After being coded, the corresponding SI bits are superimposed onto the alternative logging symbol sequences according to Equation (6). The obtained superimposed training sequence is

$$
\hat{\boldsymbol{X}}=\sqrt{\beta} \boldsymbol{S}+\sqrt{1-\beta} \boldsymbol{X}
$$

where $\beta$ is a definite power allocation factor, and $0 \leq \beta \leq$ 0.1 . The receiver makes channel estimation [31] to restore the original data information. Taking the phase sequence like Figure 3 can effectively lower the band radiation of the system and omit SI bits transmission.

At last, the processed logging signals make IFFT

Table 1. Mapping of message vector to codeword.

\begin{tabular}{ccc}
\hline Index & Message vector & Codeword \\
\hline 1 & 000 & 000000 \\
2 & 100 & 110100 \\
3 & 010 & 011010 \\
4 & 110 & 101110 \\
5 & 001 & 101001 \\
6 & 101 & 011101 \\
7 & 011 & 110011 \\
8 & 111 & 000111 \\
\hline
\end{tabular}




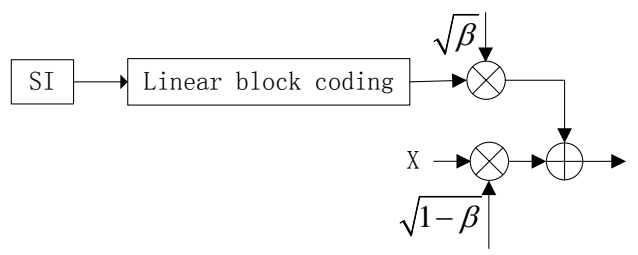

Figure 3. The procession of superimposed method.

transformation and the OFDM logging signal with the lowest PAPR is selected to transmit.

\section{The Theoretical BER Analysis of the Improved SLM Method}

The improved SLM method applies LBC to code the SI bits. It denotes 3-bits by 6-bits. Once one bit is erroneous, it will produce totally wrong receive signals. The decoding error probability of $(6,3)$ Line Block Code is:

$$
P_{M} \leq \sum_{j=t+1}^{6}\left(\begin{array}{l}
6 \\
j
\end{array}\right) p^{j}(1-p)^{6-j}
$$

Here $j=1, \cdots 2^{3}$, denotes $2^{3}$ different code words; $n=$ 6; $p$ presents transfer error probability with binary symmetrical channel. And $\left(\begin{array}{l}6 \\ j\end{array}\right)=\frac{6 !}{j !(6-j) !}$. Error-correcting capability of code is denoted by $t$,

$$
t=\left\lfloor\frac{d_{\min }-1}{2}\right\rfloor
$$

$d_{\min }$ refers to minimum distance of Line Block Code. It determines the decoding error-correcting capability of code. According to the definition, $d_{\min }$ of $(6,3) \mathrm{LBC}$ is 3.

If the bit error rate (BER) of conventional SLM is denoted by the BER of QPSK modulation $P_{Q P S K}$, the BER of the improved SLM $P$ is

$$
\begin{aligned}
& P=P_{\text {QPSK }} P_{M} \\
& P_{\text {QPSK }}=\frac{1}{2} \operatorname{erfC}\left[\sqrt{\frac{E}{2 n_{0}}}\right]
\end{aligned}
$$

It is clear that, theoretically, the BER of the improved SLM is smaller than that of the conventional SLM.

\section{Simulation Results and Analysis}

The simulation of the proposed scheme and the conventional SLM schemes is performed for the OFDM system and OFDMA downlink system specified in IEEE 802.16 standard, which uses 256 and 2048 subcarriers respectively, QPSK, and 16-QAM, and 64-QAM modulations. During this OFDM system simulation, the number of used carriers is 256 and the numbers of phase sequence are 4 and 8 . The 10000 input symbol sequences are gen- erated randomly with uniform distribution.

\subsection{The PAPR Reduction Performance Analysis of the Improved SLM Method}

Figure 4 compares the PAPR performance between proposed SLM and Coded SLM (Chen and Liang, 2007) with $u=4$, 8. Coded SLM with combining SLM and binary cyclic codes is used for both error correction and PAPR reduction. From the detailed view in Figure 4, the PAPR performance of our scheme is better than that of the traditional SLM and the Coded SLM (Chen and Liang, 2007). In the figures, "Coded SLM" denotes Coded SLM (Chen and Liang, 2007).

\subsection{The BER Performance Analysis of the Proposed SLM Method}

The channel of OFDM wireless downhole transmission systems is very complex. Simulations are carried out under the Additive White Gaussian Noise (AWGN) channel. Figure 5 compares the BER of the proposed SLM, traditional SLM and Coded SLM OFDM signals in the AWGN channel. The proposed SLM which codes SI bits of traditional SLM with linear block codes has much better BER performance than the traditional SLM method and is closer to original OFDM signals. BER of the proposed SLM with SI bits coding is a little higher than that of the Code SLM and original OFDM signals. However, the Code SLM codes all the OFDM signals which bring about very large computation. So the little scarification of BER performance is worthwhile in the proposed SLM OFDM wireless downhole transmission signals.

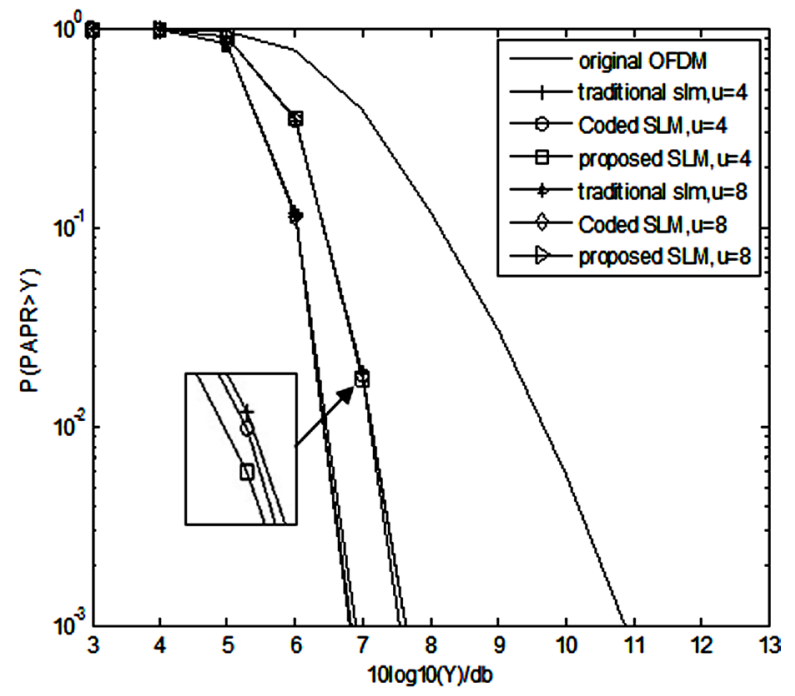

Figure 4. CCDF of the PAPR of proposed, Coded SLM (Chen and Liang, 2007) and conventional SLM scheme in OFDM wireless downhole transmission systems with $u=$ 4.8. 


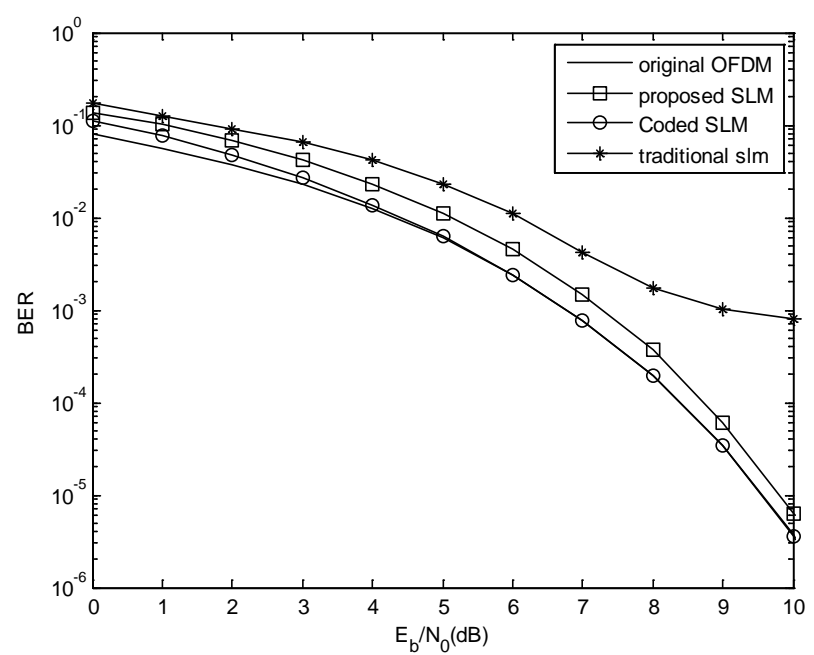

Figure 5. BER performance of the proposed, Coded SLM (Chen and Liang, 2007) and traditional SLM scheme in OFDM wireless downhole transmission systems.

\section{Conclusion}

This paper first presents the proposed OFDM wireless downhole transmission systems which are designed to provide a high-speed, real-time transmission of the downhole data. They can be promising systems to solve the bottlenecks of the existing systems. And the method of reduction PAPR in OFDM downhole transmission systems is first researched. This paper proposes a proposed SLM method using linear block codes without explicit SI and with better BER performance. It is needless to transmit and restore the side information about the phase rotation by using superimposed method. And the analysis of the BER performance analysis indicates that the proposed SLM scheme can reduce the BER. The proposed SLM scheme sacrifices a little computation because of coding, but both the encoder and decoder of linear block codes in our systems can be easily implemented.

\section{Acknowledgements}

The authors would like to thank the National Natural Science Foundation of China (No. 61072073) and the Key Project of Education Commission of Sichuan Province (No. 10ZA072) and Sichuan Province Science and Technology Support Project (No. 2012FZ0021).

\section{REFERENCES}

[1] R. Desbrandes, A. T. Bouroyne and J. A. Carter, "MWD Transmission Data Rates Can be Optimized,” Petroleum Engineer International, Vol. 59, No. 6, 1987, pp. 46-52.

[2] X. S. Liu, S. S. He and Z. C. Zhao, "Hydrodynamic Equations Model for Mud-Pulse Telemetry Transmissions,” Oil and Gas Journal, Vol. 101, No. 3, 2003, pp. 47-49.

[3] SERRA, "Fundamentals of Well-Log Interpretation,"
Schumlburger Company, 1984.

[4] Y. Cho, J. Kim, W. Yang and C. Kang, "MIMO-OFDM Wireless Communications with MATLAB,” Wiley-IEEE Press, 2010. http://dx.doi.org/10.1002/9780470825631

[5] J. Proakis and M. Salehi, "Digital Communications," 5th Edition, McGraw-Hill Companies, 2011.

[6] R. H. Carter and M. Hathaway, "Indicating and Detecting the Start of Signal Transmission Employing Frequency Division Multiplexing,” US Patent No. 20100220773, 2008.

[7] Halliburton, "LOGIQ Open-Hole Logging Platform," 2013. http://www.halliburton.com/

[8] W. X. Chen, B. B. Pei and S. Zhao, "Research of Networked Well Logging System Based on Ethernet Technology,” Well Logging Technology, Vol. 36, No. 3, 2012, pp. 286-289.

[9] J. Q. Zhang, T. Lu and Q. Li, "900kbit/s Data Transmission System Based on OFDM for Wireline Logging," Well Logging Technology, Vol. 33, No. 1, 2009, pp. 8488.

[10] R. H. Mccoy, G. L. Besser and S. D. Reed, "Systems and Methods for Downhole OFDM Communications," US Patent No. 2011047534, 2012.

[11] M. Memarzadeh, D. Johnson and U. Rice, "Maximizing the Fidelity of Log Signals Transmitted via Digital Telemetry," SPE Annual Technical Conference and Exhibition, September 2006, pp. 24-27.

[12] X. D. Wu, "A Study on High Speed Cable Telemetry Technology Based on the Multi-Carrier Modulation,” Petroleum Instruments, Vol. 25, No. 2, 2011, pp. 4-6.

[13] Y. P. Zhao, N. Li and C. Z. Wang, "Remote Wireless Transmission and Error Recovery of Log Data,” Applied Geophysics, Vol. 4, No. 5, 2007, pp. 308-312. http://dx.doi.org/10.1007/s11770-007-0042-7

[14] R. Desbrandes, "MWD Technology, Part 2 Data Transmission,” Petroleum Engineer International, Vol. 60, No. 10, 1988, pp. 48-54.

[15] R. Hutin, W. Tennent and S. V. Kashikar, "New Mud Pulse Telemetry Techniques for Deep Water Applications and Improved Real-Time Data Capabilities,” SPE/IADC 67762, 2001.

[16] W. Gravley, "Review of Downhole Measurements-While Drilling Systems. Society of Petroleum Engineer," SPE 10324, 1981.

[17] S. S. Jeng and J. M. Chen, "Efficient PAPR Reduction in OFDM Systems Based on a Companding Technique with Trapezium Distribution,” IEEE Transactions on Broadcasting, Vol. 57, No. 2, 2011, pp. 291-298. http://dx.doi.org/10.1109/TBC.2011.2112237

[18] T. Jun, P. Li and Z. H. Zhang, "Iterative Soft Compensation for OFDM Systems with Clipping and Superposition Coded Modulation," IEEE Transactions on Communications, Vol. 58, No. 10, 2010, pp. 2861-2870. http://dx.doi.org/10.1109/TCOMM.2010.083110.09296

[19] C. Nader, P. N. Landin and W. Van Moer, "Performance Evaluation of Peak-to-Average Power Ratio Reduction and Digital Re-Distortion for OFDM Based Systems," 
IEEE Transactions on Microwave Theory and Techniques, Vol. 59, No. 12, 2011, pp. 3504-3511. http://dx.doi.org/10.1109/TMTT.2011.2170583

[20] K. Hyunju, H. Eonpyo and A. Changjun, “A Pilot Symbol Pattern Enabling Data Recovery without Side Information in PTS-Based OFDM Systems," IEEE Transactions on Broadcasting, Vol. 57, No. 2, 2011, pp. 309-312.

[21] J. P. Wang, Y. Guo and X. W. Zhou, "PTS-Clipping Method to Reduce the PAPR in ROF-OFDM System," IEEE Transactions on Consumer Electronics, Vol. 55, No. 2, 2009, pp. 356-359. http://dx.doi.org/10.1109/TCE.2009.5174393

[22] O. Daoud and O. Alani, "Reducing the PAPR by Utilisation of the LDPC Code," IET Communications, Vol. 3, No. 4, 2009, pp. 520-529. http://dx.doi.org/10.1049/iet-com.2008.0344

[23] M. Sabbaghian, Y. J. Kwak and B. Smida, "Near Shannon Limit and Low Peak to Average Power Ratio Turbo Block Coded OFDM," IEEE Transactions on Communications, Vol. 59, No. 8, 2011, pp. 2042-2045. http://dx.doi.org/10.1109/TCOMM.2011.080111.090356

[24] M. Sabbaghian, K. Yongjun and V. Tarokh, "New Codes from Dual BCH Codes with Applications in Low PAPR OFDM," IEEE Transactions on Wireless Communications, Vol. 10, No. 12, 2011, pp. 3990-3994. http://dx.doi.org/10.1109/TWC.2011.100611.101388

[25] D. W. Lim, H. S. Noh and H. B. Jeon, "Multi-Stage TR Scheme for PAPR Reduction in OFDM Signals," IEEE Transactions on Broadcasting, Vol. 55, No. 2, 2009, pp. 300-304. http://dx.doi.org/10.1109/TBC.2009.2013988
[26] H. S. Chen and H. Y. Liang, "Combined Selective Mapping and Binary Cyclic Codes for PAPR Reduction in OFDM Systems,” IEEE Transactions on Wireless Communications, Vol. 6, No. 10, 2007, pp. 3524-3528. http://dx.doi.org/10.1109/TWC.2007.060145

[27] S. S. Eom, H. Nam and Y. C. C. Ko, "Low-Complexity PAPR Reduction Scheme without Side Information for OFDM Systems," IEEE Transactions on Signal Processing, Vol. 60, No. 7, 2012, pp. 3657-3669. http://dx.doi.org/10.1109/TSP.2012.2191779

[28] M. F. Naeiny and F. Marvasti, "Selected Mapping Algorithm for PAPR Reduction of Space-Frequency Coded OFDM Systems without Side Information,” IEEE Transactions on Vehicular Technology, Vol. 60, No. 3, 2011, pp. 1211-1216. http://dx.doi.org/10.1109/TBC.2011.2151570

[29] H. B. Jeon, J. S. No and D. J. Shin, “A Low-Complexity SLM Scheme Using Additive Mapping Sequences for PAPR Reduction of OFDM Signals," IEEE Transactions on Broadcasting, Vol. 57, No. 4, 2011, pp. 866-875. http://dx.doi.org/10.1109/TBC.2011.2151570

[30] N. Chen and G. T. Zhou, "Superimposed Training for OFDM: A Peak-to-Average Power Ratio Analysis,” IEEE Transactions on Signal Processing, Vol. 54, No. 6, 2006, pp. 2277-2287. http://dx.doi.org/10.1109/TSP.2006.874299

[31] R.-Z. Luo, N. Chen and G.-T. Zhou, "Channel Estimation Scheme Using Superimposed Training Sequences with Low Power," Acta Electronica Sinica, Vol. 36, No. 1, 2008, pp. 177-179. 